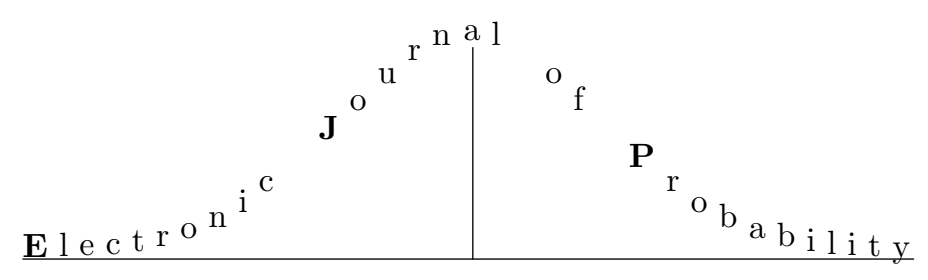

Vol. 11 (2006), Paper no. 26, pages 670-685.

Journal URL

http://www.math.washington.edu/ ejpecp/

\title{
Behavior of a second class particle in Hammersley's process
}

\author{
Eric Cator and Sergei Dobrynin \\ Delft Institute of Applied Mathematics \\ Delft University of Technology \\ Mekelweg 4, 2628 CD Delft \\ The Netherlands \\ e.a.cator@ewi.tudelft.nl
}

\begin{abstract}
In the case of a rarefaction fan in a non-stationary Hammersley process, we explicitly calculate the asymptotic behavior of the process as we move out along a ray, and the asymptotic distribution of the angle within the rarefaction fan of a second class particle and a dual second class particle. Furthermore, we consider a stationary Hammersley process and use the previous results to show that trajectories of a second class particle and a dual second class particles touch with probability one, and we give some information on the area enclosed by the two trajectories, up until the first intersection point. This is linked to the area of influence of an added Poisson point in the plane
\end{abstract}

Key words: Hammersley's process, second class particles, rarefaction fan

AMS 2000 Subject Classification: Primary 60C05,60K35; Secondary: 60F05.

Submitted to EJP on May 23 2006, final version accepted June 212006. 


\section{Introduction}

In [HAmmersley (1972)], a discrete interacting particle process is introduced to study the behavior of the length of longest increasing subsequences of random permutations. In [Aldous And Diaconis (1995)], this discrete process is generalized to a continuous time interacting particle process on the real line, and they use the ergodic decomposition theorem to show local convergence to a Poisson process, when moving out along a ray. In this paper, we will consider Hammersley's process with sources and sinks, as introduced in [GroenEBoom (2002)]. For an extensive description of this process, we refer to [CATor And Groeneboom (2005)], since our results will be partly based on results derived in that paper. Here we will suffice with a brief description, based on Figure 1.

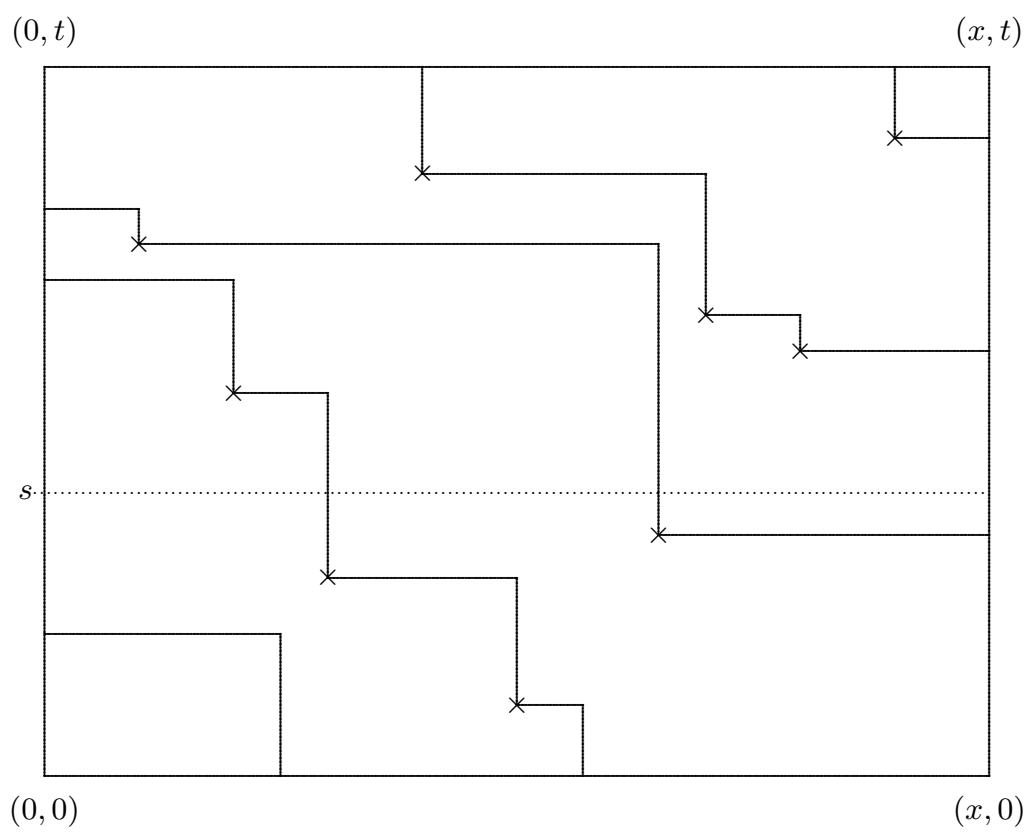

Figure 1: Space-time paths of the Hammersley's process, with sources and sinks.

We consider the space-time paths of particles that started on the $x$-axis as sources, distributed according to a Poisson distribution and we consider the $t$-axis as a time axis. In the positive quadrant we have a Poisson process of what we call $\alpha$-points (denoted in Figure 1 by $\times$ ). At the time an $\alpha$-point appears, the particle immediately to the right of it jumps to the location of the $\alpha$-point. Finally, we have a Poisson process of sinks on the $t$-axis. Each sink makes the leftmost particle disappear. All three Poisson processes are assumed to be independent. To know the particle configuration at time $s$, we intersect a line at time $s$ with the space-time paths.

In [CAtor And Groeneboom (2005)], a connection was made between the continuous time Hammersley process and the behavior of second class particles, which are well studied in the literature on discrete interacting particle systems such as TASEP; see for example [LIGGETT (1999)]. For the Hammersley process, it is natural to consider two types of second class particles: the 
usual one, where one adds an extra particle at the origin, and a dual second class particle, which corresponds to adding an extra sink at the origin (or removing the leftmost particle). In fact, the trajectories of these two particles correspond to the two longest paths of the timereversed process such that all possible longest paths fall between these two longest paths. We can study the trajectories of these two particles at the same time, that is, for one realization of the Hammersley process. In this paper we study the behavior of a second class particle and its dual particle in the case of a rarefaction fan, a phenomenon often observed in interacting particle systems. In [FERRARI AND KIPNIS (1995)] this problem is considered for TASEP. In [SEPp ÄLÄINEN (2002)] the Hammersley process is considered with general initial conditions, but the rarefaction fan is not treated. Also, our methods are quite different and build more on the ideas of [Cator and Groeneboom (2005)]. Around the time this paper was written, the preprint [Colleti And Pimentel (2006)] appeared, where the asymptotic distribution of the angle of the (dual) second class particle is also calculated (see Theorem 2.5), but not by proving the asymptotic behavior of the Hammersley process in the rarefaction fan when moving out along a ray (Theorem 2.2). They do state that the trajectory of a (dual) second class particle will almost surely converge to a (random) line starting at the origin, relying on results obtained by Baik and Raines using the RSK machinery. In the final section we will study the interaction between a second class particle and its dual in the case of a stationary Hammersley process, and show that they will touch with probability one. This should not be confused with the situation where we have two second class particles, since the dual second class particle has different behavior from a "normal" second class particle. We also study the area between the two trajectories up until this point of touch. We did not find results in the literature on discrete interacting particle systems that were similar to the results of our last section, so this interaction phenomenon may be a specific feature of the Hammersley process.

\section{Second class particles in a rarefaction fan}

Let $\lambda, \mu$ be two positive reals, such that $\lambda \mu<1$. Let $t \mapsto L_{\lambda, \mu}(\cdot, t)$ be Hammersley's process developing in time $t$, generated by a Poisson process of sources on the positive x-axis of intensity

$\lambda$, a Poisson process of sinks on the time axis of intensity $\mu$ and a Poisson process on $\mathbb{R}_{+}^{2}$ of intensity 1 , where these Poisson processes are independent. Here, $L_{\lambda, \mu}(\cdot, t)$ signifies the counting process that counts the number of Hammersley particles on the half-line $(0, \infty) \times\{t\}$. As was shown in [Groeneboom (2002)], the case $\lambda \mu=1$ corresponds to a stationary Hammersley process, which means that for each $t \geq 0, L_{\lambda, \mu}(\cdot, t)$ is a Poisson process with intensity $\lambda$.

As we mentioned in the introduction, we will consider two kinds of second class particles. A "normal" second class particle is created by putting an extra source in the origin. A dual second class particle is created by putting an extra sink in the origin. The trajectories $\left(X_{t}, t\right)$ of a second class particle and $\left(X_{t}^{\prime}, t\right)$ of a dual second class particle are shown in Figure 2. Note that we always have $X_{t}^{\prime} \geq X_{t}$.

Now consider the reversed process, where we use the North exits through $[0, x] \times\{t\}$ as sources, the East exits through $\{x\} \times[0, t]$ as sinks and the $\beta$-points (these are the upper-right corners of the space-time paths) as our Poisson process in $[0, x] \times[0, t]$. Burke's Theorem for Hammersley's process (see [Cator and Groeneboom (2005)]) shows that this process is again a stationary Hammersley process, if we start with a stationary process. It is not hard to see from Figure 2 


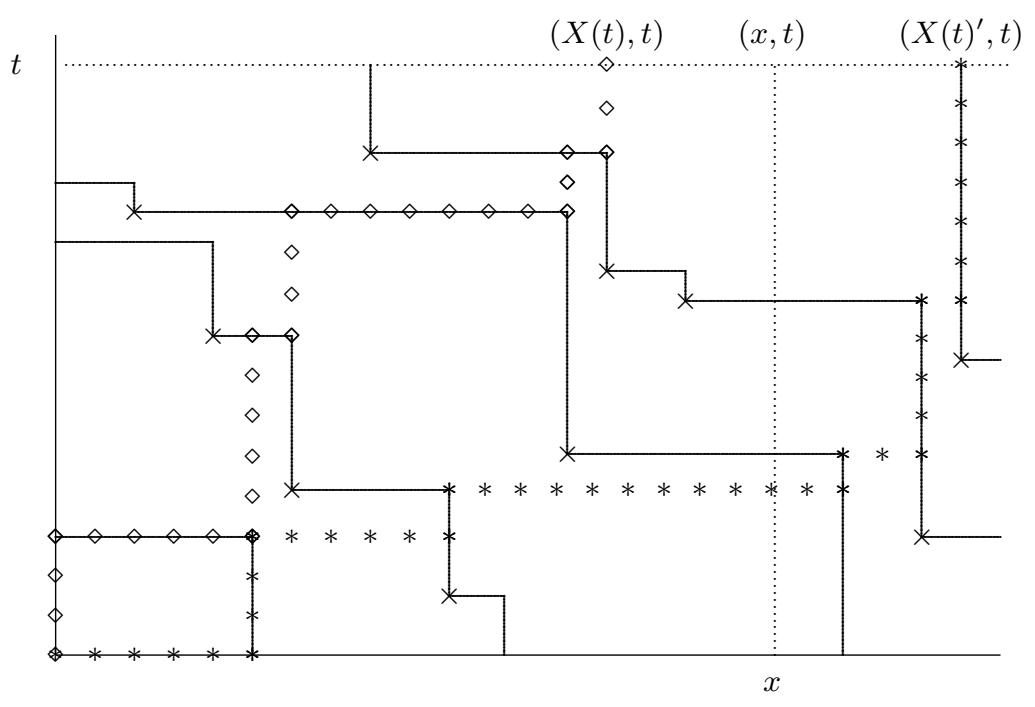

Figure 2: Trajectories of $\left(X_{t}, t\right)$ and $\left(X_{t}^{\prime}, t\right)$

that the trajectories of $X$ and $X^{\prime}$ correspond to longest paths from $(x, t)$ to $(0,0)$ in the reversed process.

A second class particle and a dual second class particle are symmetrical with respect to the main diagonal in the following sense: the reflected trajectory of a dual second class particle is a trajectory of a "normal" second class particle for the reflected realization, that is, with the sources and sinks intensity exchanged.

A very important property of second class and dual second class particles is given in the following lemma, which is slightly more general than Lemma 2.2 in [CATor And Groeneboom (2005)], but the proofs of these two lemma's are very similar, and therefore omitted here.

Lemma 2.1 Consider a Hammersley process $L$ with some configuration of sources and sinks. Define two coupled Hammersley processes $\bar{L}$ and $\underline{L}$ such that all three processes use the same $\alpha$-points. Furthermore, $\bar{L}$ uses the same sources as $L$, but only a subset of the sinks of $L$, whereas $\underline{L}$ uses the same sinks as $L$, but only a subset of the sources of $L$. Denote with $X$ and $X^{\prime}$ a second class particle respectively a dual second class particle for the process $L$. Then the space-time paths of $L$ and $\bar{L}$ coincide below the trajectory of $X$, whereas the space-time paths of $L$ and $\underline{L}$ coincide above the trajectory of $X^{\prime}$.

The reader can verify this lemma by looking at Figure 2 .

The results in this section are also valid when $\lambda$ and/or $\mu$ are equal to 0 , if we keep in mind that whenever $\lambda=0$, a dual second class particle remains on the $x$-axis (so $X_{t}^{\prime}=+\infty$ for $t>0$ ), whereas when $\mu=0$, a second class particle remains on the $t$-axis (so $X_{t}=0$ for $t>0$ ).

For $\rho>0$, we denote by $t \mapsto L_{\rho}(\cdot, t)$ a stationary Hammersley process, generated by a process of sources of intensity $\rho$, a process of sinks of intensity $1 / \rho$ and a process of $\alpha$-points of intensity 1.

Throughout this section we are interested in the asymptotic behavior of the non-stationary 
process $L_{\lambda, \mu}$ and trajectories of its second class and dual second class particles. We recall here the corresponding results for a stationary process, obtained in [CATor And Groeneboom (2005)].

Theorem 2.1 (Cator and Groeneboom) Let $t \rightarrow L_{\rho}(\cdot, t)$ be a stationary Hammersley process with intensity of sources $\rho$ and intensity of sinks $1 / \rho$. Let $X_{t}$ be the $x$-coordinate of a second class particle at time $t$ and $X_{t}^{\prime}$ be the $x$-coordinate of a dual second class particle at time $t$.

Then:

(i) $t^{-1} X_{t} \rightarrow 1 / \rho^{2}$ almost surely as $t \rightarrow \infty$

(ii) $t^{-1} X_{t}^{\prime} \rightarrow 1 / \rho^{2}$ almost surely as $t \rightarrow \infty$

This theorem combines the results of Theorem 2.1 and Remark 2.1 of [Cator and Groeneboom (2005)]. We can now show the following theorem, describing the asymptotic local intensities of the process $L_{\lambda, \mu}$ when moving out along a ray $t=a x$, for $a>0$.

Theorem 2.2 Let $a>0$. Consider the random particle configuration with counting process

$$
y \mapsto L_{\lambda, \mu}(x+y, a x)-L_{\lambda, \mu}(x, a x), y \geq-x .
$$

Then:

(i) If $a>1 / \mu^{2}$, the process converges in distribution, as $x \rightarrow \infty$, to a homogeneous Poisson process on $\mathbb{R}$, with intensity $1 / \mu$.

(ii) If $1 / \mu^{2}>a>\lambda^{2}$, the process converges in distribution, as $x \rightarrow \infty$, to a homogeneous Poisson process on $\mathbb{R}$, with intensity $\sqrt{a}$.

(iii) If $\lambda^{2}>a$, the process converges in distribution, as $x \rightarrow \infty$, to a homogeneous Poisson process on $\mathbb{R}$, with intensity $\lambda$.

\section{Proof:}

(i) We couple the process $L_{\lambda, \mu}$ with a stationary process $L_{1 / \mu}$ via the same realization of the Poisson process in $\mathbb{R}_{+}^{2}$, the same realization of sinks on the $t$-axis, and a "thickened" set of sources. This means that we independently add to the Poisson process of intensity $\lambda$ on the $x$ axis a Poisson process of intensity $1 / \mu-\lambda$. This way of coupling (when only one set is changed (thinned or thickened) and the two others are kept fixed) will be used throughout the paper without further explanation.

Let $\left(X_{t}^{\prime}, t\right)$ be the trajectory of an isolated dual second class particle at zero of the process $L_{1 / \mu}$. As the process $L_{\lambda, \mu}$ can be obtained from $L_{1 / \mu}$ by removing the added sources, the space-time paths of both processes coincide above the trajectory $\left(X_{t}^{\prime}, t\right)$ (Lemma 2.1). Now it is enough to apply Theorem 2.1, stating that $X_{t}^{\prime} / t$ almost surely converges to $\mu^{2}$, and to use the stationarity of $L_{1 / \mu}$.

(ii) This proof is analogous to the proof of Corollary 2.1, (i), in [CAtor and Groeneboom (2005)]. Let $a^{\prime}$ be such that $1 / \mu^{2}>a^{\prime}>a$. Note that $\sqrt{a^{\prime}}>\lambda$ and $1 / \sqrt{a^{\prime}}>\mu$. Now we couple the process $L_{\lambda, \mu}$ with a process $L_{\sqrt{a^{\prime}}}$ generated by the same realization of points on the plane and thickened sets of sources and sinks of intensities $\sqrt{a^{\prime}}$ and $1 / \sqrt{a^{\prime}}$ correspondingly. 


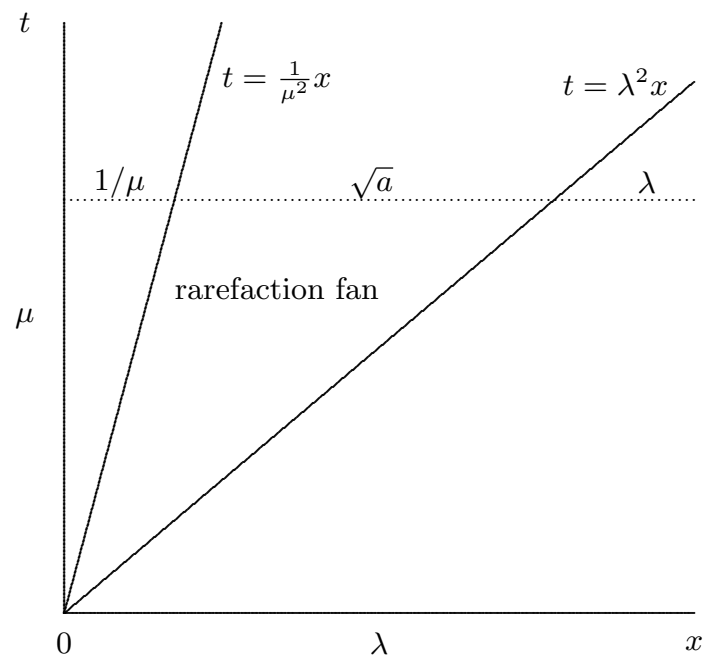

Figure 3: Illustration of Theorem 2.2

Below the trajectory $\left(X_{t}, t\right)$ of an isolated second class particle at zero of $L_{\sqrt{a^{\prime}}}$, the space-time paths of $L_{\sqrt{a^{\prime}}}$ coincide with the space-time paths of the process $\bar{L}_{\sqrt{a^{\prime}}}$, which is obtained by removing all sinks (again Lemma 2.1).

Note that for any $t$, the set of intersections of space-time paths of the process $L_{\lambda, \mu}$ with the horizontal level $t$ is contained in the set of intersection of space-times paths of $\bar{L} \sqrt{a^{\prime}}$ with the horizontal level $t$. In other words, at all times the process $\bar{L}_{\sqrt{a^{\prime}}}$ contains the particles of $L_{\lambda, \mu}$, and may have more particles, since the first process has more sources and less sinks. It follows that the set of intersections of space-time paths of $L_{\lambda, \mu}$ with any horizontal interval lying to the right of the trajectory $\left(X_{t}, t\right)$ is contained in the set of intersections of paths of $L_{\sqrt{a^{\prime}}}$ with this interval.

Similarly, we consider $a^{\prime \prime}$ such that $a>a^{\prime \prime}>\lambda^{2}$ and couple $L_{\lambda, \mu}$ with $L_{\sqrt{a^{\prime \prime}}}$. Now we have that above the trajectory $\left(X_{t}^{\prime}, t\right)$ of an isolated dual second class particle at zero of $L_{\sqrt{a^{\prime \prime}}}$, the space-time paths of $L_{\sqrt{a^{\prime \prime}}}$ coincide with the paths of $L_{\sqrt{a^{\prime \prime}}}$, which is obtained by removing all sources. This time we conclude that the set of intersections of space-time paths of $L_{\lambda, \mu}$ with any horizontal interval lying to the left of the trajectory $\left(X_{t}^{\prime}, t\right)$ contains the set of intersections of paths of $L_{\sqrt{a^{\prime \prime}}}$ with this interval.

The situation is now as follows: if we fix $R>0$ and consider the process $L_{\lambda, \mu}$ on the horizontal interval $[x-R, x+R] \times\{a x\}$, we know that for any fixed $a^{\prime}$ and $a^{\prime \prime}$ such that $1 / \mu^{2}>a^{\prime}>a>a^{\prime \prime}>\lambda^{2}$ and for $x$ big enough, the particles of this process are a subset of the stationary process $L_{\sqrt{a^{\prime}}}$ and a superset of the stationary process $L_{\sqrt{a^{\prime \prime}}}$. Now we are exactly in the situation of the proof of the Corollary 2.1, (i), [CATor And Groeneboom (2005)] and statement (ii) follows by considering the Laplace transform of the number of particles in fixed disjoint intervals and using dominated convergence.

(iii) The proof of the last statement proceeds along the same lines as the proof of statement (i). Couple $L_{\lambda, \mu}$ with $L_{\lambda}$, note that their space-time paths coincide below the trajectory $\left(X_{t}, t\right)$ 
of an isolated second class particle at zero of the process $L_{\lambda}$ and use Theorem 2.1 and the stationarity of $L_{\lambda}$.

Let $F_{\lambda, \mu}(x, t)$ be the flux of the process $L_{\lambda, \mu}$ at $(x, t)$, which we define by the number of spacetime paths of $L_{\lambda, \mu}$ contained in the rectangle $[0, x] \times[0, t]$. Note that $F_{\lambda, \mu}(x, t)$ can be represented as the number of crossings of the interval $[0, x] \times\{t\}$ by space-time paths of $L_{\lambda, \mu}$ plus the number of crossings of the interval $\{0\} \times[0, t]$. It is also equal to the number of crossings of the interval $[0, x] \times\{0\}$ plus the number of crossings of the interval $\{x\} \times[0, t]$ by space-time paths of $L_{\lambda, \mu}$.

Definition 2.3 When moving out along a ray $t=a x$, we define the local intensity function $I(x, h)$ by the following formula:

$$
I(x, h)=\mathbb{E}\left(F_{\lambda, \mu}(x+h, a x)-F_{\lambda, \mu}(x, a x)\right) .
$$

We suppress the dependence on $a, \lambda$ and $\mu$ in our notation.

Note that

$$
I(x, h)=\mathbb{E}\left(L_{\lambda, \mu}(x+h, a x)-L_{\lambda, \mu}(x, a x)\right),
$$

so it is equal to the expected number of crossings of the interval $[x, x+h] \times\{a x\}$ by space-time paths of the process $L_{\lambda, \mu}$.

Corollary 2.4 Fix the positive constants $a, \lambda$ and $\mu$. We have

$$
\lim _{x \rightarrow \infty} I(x, h)= \begin{cases}h / \mu, & a \geq 1 / \mu^{2} \\ \sqrt{a} \cdot h, & 1 / \mu^{2}>a \geq \lambda^{2} \\ \lambda \cdot h, & \lambda^{2}>a \geq 0 .\end{cases}
$$

Proof: Coupling in a standard way the process $L_{\lambda, \mu}$ with the process $L_{1 / \mu}$ (adding sources), we can estimate

$$
L_{\lambda, \mu}(x+h, a x)-L_{\lambda, \mu}(x, a x) \leq L_{1 / \mu}(x+h, a x)-L_{1 / \mu}(x, a x)
$$

for any realization and any $x \geq 0$. Define for $x \geq 0$

$$
A_{x}=L_{\lambda, \mu}(x+h, a x)-L_{\lambda, \mu}(x, a x) \text { and } B_{x}=L_{1 / \mu}(x+h, a x)-L_{1 / \mu}(x, a x) .
$$

Define a random variable $A$, whose distribution depends on the fixed parameters $a, \lambda$ and $\mu$, as follows:

$$
A \sim \begin{cases}\operatorname{Pois}(h / \mu), & a \geq 1 / \mu^{2}, \\ \operatorname{Pois}(\sqrt{a} \cdot h), & 1 / \mu^{2}>a \geq \lambda^{2} \\ \operatorname{Pois}(\lambda \cdot h), & \lambda^{2}>a \geq 0 .\end{cases}
$$

Theorem 2.2 shows that

$$
A_{x} \stackrel{\mathrm{d}}{\longrightarrow} A(x \rightarrow \infty)
$$


Furthermore,

$$
\forall x \geq 0: B_{x} \sim \operatorname{Pois}(h / \mu)
$$

For any $M>0$ we have

$$
\mathbb{E}\left(A_{x}\right)=\mathbb{E}\left(A_{x} \wedge M\right)+\mathbb{E}\left(\left(A_{x}-M\right) 1_{\left\{A_{x}>M\right\}}\right) .
$$

Also,

$$
\forall x>0: \mathbb{E}\left(\left(A_{x}-M\right) 1_{\left\{A_{x}>M\right\}}\right) \leq \mathbb{E}\left(\left(B_{x}-M\right) 1_{\left\{B_{x}>M\right\}}\right)=\mathbb{E}\left(\left(B_{0}-M\right) 1_{\left\{B_{0}>M\right\}}\right) .
$$

Since

$$
\lim _{x \rightarrow \infty} \mathbb{E}\left(A_{x} \wedge M\right)=\mathbb{E}(A \wedge M), \quad \lim _{M \rightarrow \infty} \mathbb{E}(A \wedge M)=\mathbb{E} A
$$

and

$$
\lim _{M \rightarrow \infty} \mathbb{E}\left(\left(B_{0}-M\right) 1_{\left\{B_{0}>M\right\}}\right)=0,
$$

we conclude that

$$
\lim _{x \rightarrow \infty} \mathbb{E}\left(A_{x}\right)=\mathbb{E} A
$$

In the next theorem we will discuss the behavior of $X_{t}$ and $X_{t}^{\prime}$, the second class particle and the dual second class particle of the process $L_{\lambda, \mu}$.

Theorem 2.5 Let $L_{\lambda, \mu}$ be the non-stationary Hammersley process defined above. Let $X_{t}$ be the $x$-coordinate at time $t$ of a second class particle starting at zero. Then

$$
\lim _{x \rightarrow \infty} P\left(X_{a x}>x\right)= \begin{cases}1, & a \geq 1 / \mu^{2}, \\ \frac{\sqrt{a}-\lambda}{1 / \mu-\lambda,} & 1 / \mu^{2}>a \geq \lambda^{2} \\ 0, & \lambda^{2}>a \geq 0 .\end{cases}
$$

Proof: To prove this theorem we will calculate the derivative $\left.\frac{\partial}{\partial h} I(x, h)\right|_{h=0}$. The proof is inspired by the proof of Theorem 1, [FERRARI AND KIPNIS (1995)], where the analogous problem for TASEP is solved.

Fix $t$ and $x$ and consider some small $h>0$. We couple the process $L_{\lambda, \mu}$ with a process $\hat{L}_{\lambda, \mu}$ constructed in the following way. Consider the same Poisson realizations of points in the plane. Consider the same realization of sinks. Consider the same realization of sources on the interval $(h, \infty) \times\{0\}$. On the interval $[0, h] \times\{0\}$ we add an independent Poisson process of sources of intensity $1 / \mu-\lambda$. The situation is illustrated in Figure 4.

Note that the process $\hat{L}_{\lambda, \mu}$, restricted to the rectangle $[0, h] \times[0, t]$, is a stationary Hammersley process with intensity of sources $1 / \mu$ and intensity of sinks $\mu$. Thus, the set of crossings of spacetime trajectories of $\hat{L}_{\lambda, \mu}$ with the vertical segment $\{h\} \times[0, t]$ is a Poisson process of intensity $\mu$, independent of the sources on $(h, \infty) \times\{0\}$ and of the Poisson points in $(h, \infty) \times[0, t]$ (this follows from the stationarity of the Hammersley process when viewed from left to right). Now it follows that the number of space-time paths of the process $\hat{L}_{\lambda, \mu}$ inside the rectangle $[h, x+h] \times[0, t]$ 


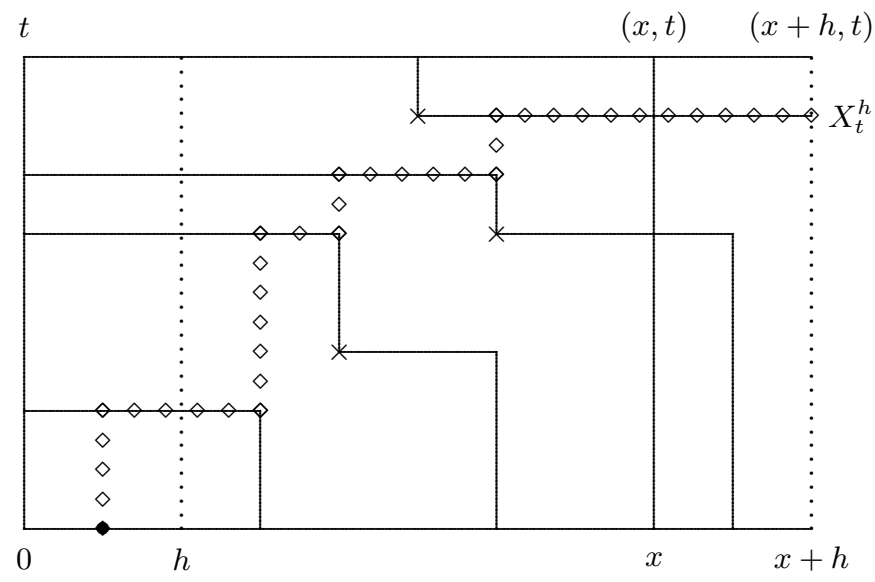

Figure 4: trajectory $X_{t}^{h}$ of added point $(\bullet)$ hits the right side and the flux $\bar{F}_{\lambda, \mu}(x, t)$ is decreased; for this realization it decreases from 4 to 3 .

(which we will denote by $\hat{F}_{\lambda, \mu}(x, t)$ ), has the same distribution as the flux of the process $L_{\lambda, \mu}$ on the rectangle $[0, x] \times[0, t]$.

We are interested in the difference

$$
F_{\lambda, \mu}(x+h, t)-\hat{F}_{\lambda, \mu}(x, t)
$$

This difference is equal to the difference between the number of space-time paths of the process $L_{\lambda, \mu}$ in the rectangle $[0, x+h] \times[0, t]$ and the number of space-time paths of the process $\hat{L}_{\lambda, \mu}$ in the rectangle $[h, x+h] \times[0, t]$.

As we are interested in the expectation of this difference only up to the first order of $h$ when $h \downarrow 0$, we can distinguish two cases. In the first case there is no extra source in $[0, h] \times\{0\}$, which means that the space-time paths of $L_{\lambda, \mu}$ and $\hat{L}_{\lambda, \mu}$ are the same. In the second case there is an extra source in $[0, h] \times\{0\}$.

In the first case the difference (2.1) is equal to the number of sources of $L_{\lambda, \mu}$ in $[0, h] \times\{0\}$, since those sources are not counted in $\hat{F}_{\lambda, \mu}(x, t)$. Clearly, the expected number of sources in $[0, h] \times\{0\}$ equals $\lambda h$.

In the second case we have to be more careful. First of all, the probability of having an extra source equals, in first order, $(1 / \mu-\lambda) h$. The added source can affect the space-time paths of the original $L_{\lambda, \mu}$ inside the rectangle $[h, x+h] \times[0, t]$ and can change the flux. Note that the change in the space-time paths will happen above the trajectory of the added point, considered as a second-class particle. If this trajectory hits the right side of the rectangle (i.e. $\{x+h\} \times[0, t]$ ), it follows that one of the intersections of space-time paths of $L_{\lambda, \mu}$ with the right side is eliminated in $\left.\bar{L}_{(} \lambda, \mu\right)$; see Figure 4. Thus, in this case the difference (2.1) is +1 . But if the trajectory of the added point (which we define by $X_{t}^{h}$ ) does not hit the vertical segment $\{x+h\} \times[0, t]$, it follows that the sets of intersections are the same for both processes and the difference is zero again. 
Now we can conclude that

$$
\mathbb{E}\left(F_{\lambda, \mu}(x+h, t)-F_{\lambda, \mu}(x, t)\right)=\lambda h+(1 / \mu-\lambda) h P\left(X_{t}^{h}>x+h\right)+O\left(h^{2}\right) .
$$

Taking $t=a x$, dividing by $h$ and taking the limit $h \rightarrow 0$ we have

$$
\left.\frac{\partial}{\partial h} I(x, h)\right|_{h=0}=\lambda+(1 / \mu-\lambda) P\left(X_{a x}>x\right) .
$$

From this it follows that $\frac{\partial I(x, h)}{\partial h}$ is non-increasing in $h \in[-1,1]$ for any fixed $x \geq 1$. Indeed, fix $h_{0} \in[-1,1]$. Use $(2.2)$ to conclude that

$$
\left.\frac{\partial}{\partial h} I(x, h)\right|_{h=h_{0}}=\left.\frac{\partial}{\partial h} I\left(x+h_{0}, h\right)\right|_{h=0}=\lambda+(1 / \mu-\lambda) P\left(X_{a x}>x+h_{0}\right) .
$$

The probability $P\left(X_{a x}>x+h_{0}\right)$ obviously does not increase in $h_{0}$.

We are now in the following situation: we have a family $\{I(x, h): x \geq 1\}$ of functions of $h$, where $h \in[-1,1]$. For all $x$ the function $I(x, h)$ is differentiable on $[-1,1]$ and concave. Moreover, we know that the family $I(x, h)$ has a pointwise limit, which is also differentiable on $[-1,1]$ (Corollary 2.4). It follows that the family of derivatives also converges to the derivative of the limit, in other words

$$
\lim _{x \rightarrow \infty} \lim _{h \rightarrow 0} \frac{1}{h} I(x, h)=\lim _{h \rightarrow 0} \lim _{x \rightarrow \infty} \frac{1}{h} I(x, h) .
$$

This is a standard result from analysis.

It follows that

$$
\lambda+(1 / \mu-\lambda) \lim _{x \rightarrow \infty} P\left(X_{a x}>x\right)= \begin{cases}1 / \mu, & a \geq 1 / \mu^{2} \\ \sqrt{a}, & 1 / \mu^{2}>a \geq \lambda^{2} \\ \lambda, & \lambda^{2}>a \geq 0\end{cases}
$$

which finishes the proof of the theorem.

Corollary 2.6 Let $L_{\lambda, \mu}$ be the non-stationary Hammersley process defined above. Let $X_{t}^{\prime}$ be the $x$-coordinate at time $t$ of a dual isolated second class particle at zero. Then

$$
\lim _{x \rightarrow \infty} P\left(X_{a x}^{\prime}>x\right)= \begin{cases}1, & a \geq 1 / \mu^{2} \\ \frac{1 / \lambda-\sqrt{1 / a}}{1 / \lambda-\mu,} & 1 / \mu^{2}>a \geq \lambda^{2} \\ 0, & \lambda^{2}>a \geq 0 .\end{cases}
$$

Proof: This follows from the symmetry of the trajectories of a second class particle and a dual second class particle (reversing the role of $x$ and $t$, interchanging $\lambda$ and $\mu$ and replacing $a$ by $1 / a)$. 


\section{Second class particles and dual second class particles in the stationary case}

Now we will turn our attention to the stationary case. Let $\lambda>0$ and consider the Hammersley process $L_{\lambda}$ with intensity of sources $\lambda$, intensity of sinks $1 / \lambda$ and intensity of $\alpha$-points 1 . As it follows from [Cator and Groeneboom (2005)] (see also Theorem 2.1), the trajectories of a second class particle at zero, $\left(X_{t}, t\right)$, and of a dual second class particle, $\left(X_{t}^{\prime}, t\right)$, both asymptotically tend to the ray $t=\lambda^{2} x$. We will prove that these trajectories intersect with probability one and provide some information on the area enclosed by these trajectories, the origin and the first intersection point.

Theorem 3.1 Let $L_{\lambda}$ be the stationary Hammersley process defined above and let $\left(X_{t}, t\right)$ and $\left(X_{t}^{\prime}, t\right)$ be the trajectories of a second class and a dual second class particle respectively. Then $\left(X_{t}, t\right)$ and $\left(X_{t}^{\prime}, t\right)$ intersect with probability one.

Proof: To prove this theorem we will again use coupling. Consider the standard coupling of $L_{\lambda}$ with $L_{\lambda, 0}$ by removing all sinks. The space-time paths of $L_{\lambda}$ and $L_{\lambda, 0}$ coincide strictly below the trajectory $\left(X_{t}, t\right)$ (Lemma 2.1). Choose a realization such that $\left(X_{t}^{\prime}, t\right)$ tends to the ray $t=\lambda^{2} x$. If $\left(X_{t}^{\prime}, t\right)$ and $\left(X_{t}, t\right)$ do not intersect for this realization, then the trajectory of a dual second class particle of the process $L_{\lambda, 0}$ coincides with $\left(X_{t}^{\prime}, t\right)$ and, consequently, will also tend to the ray $t=\lambda^{2} x$. Suppose this event has probability $\varepsilon>0$. Denote $X_{0}^{\prime}(t)$ as the $x$-coordinate at time $t$ of a dual second class particle for the process $L_{\lambda, 0}$. The previous discussion shows that

$$
P\left(\lim _{x \rightarrow \infty} X_{0}^{\prime}\left(\lambda^{2} x\right) / x=1\right) \geq \varepsilon .
$$

Then for any $a>\lambda^{2}$, we would have

$$
P\left(\exists M: \forall x \geq M: X_{0}^{\prime}\left(\lambda^{2} x\right)>\frac{\lambda^{2}}{a} x\right) \geq \varepsilon
$$

This implies that there exists $M>0$ such that

$$
P\left(\forall x \geq M: X_{0}^{\prime}\left(\lambda^{2} x\right)>\frac{\lambda^{2}}{a} x\right) \geq \varepsilon / 2 .
$$

This in turn implies

$$
\forall x \geq M: P\left(X_{0}^{\prime}\left(\lambda^{2} x\right)>\frac{\lambda^{2}}{a} x\right) \geq \varepsilon / 2 \quad \Leftrightarrow \quad \forall y \geq \frac{a}{\lambda^{2}} M: P\left(X_{0}^{\prime}(a y)>y\right) \geq \varepsilon / 2
$$

This contradicts Corollary 2.6 for a close enough to $\lambda^{2}$, which proves that $\left(X_{t}, t\right)$ and $\left(X_{t}^{\prime}, t\right)$ intersect with probability one.

Now we will study the expected area enclosed by the trajectories of a second class and a dual second class particle. The main idea comes from the following observation: suppose we have a realization of a stationary Hammersley process in the positive quadrant. Now we add an extra 

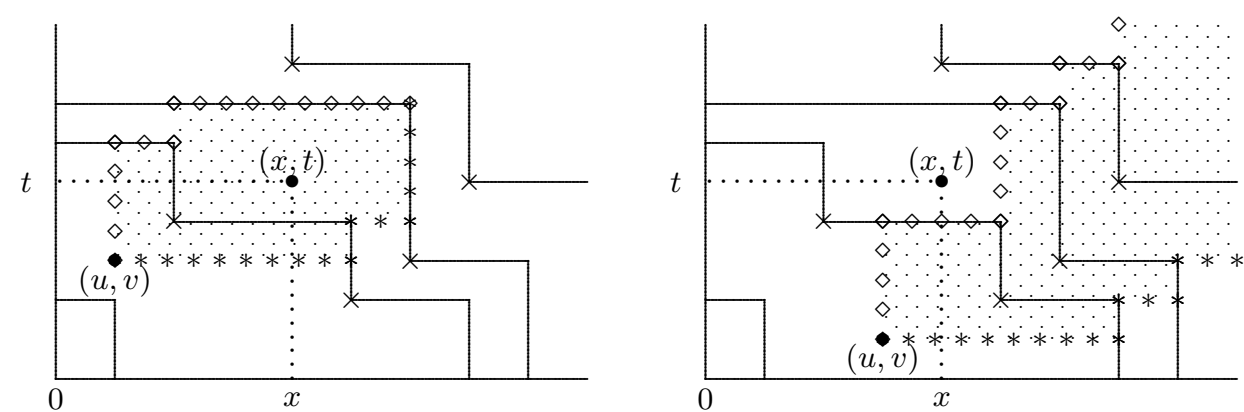

Figure 5: In the left picture, the flux at the point $(x, t)$ increases, for this realization from 2 to 3 . In the right picture, the flux at $(x, t)$ remains the same, for this realization equal to 2 .

$\alpha$-point at $(u, v)$. How does this change the space-time paths? The situation is depicted in Figure 5.

Adding an extra $\alpha$-point at $(u, v)$ moves the first particle immediately to the right of $(u, v)$ to $(u, v)$; this corresponds to a dual second class particle that starts at $(u, v)$. However, since there is now a particle at $(u, v)$, the first "sink", or crossing on $\{u\} \times(v, \infty)$, also disappears, which corresponds to a "normal" second class particle that starts at $(u, v)$. Within the region, enclosed by the trajectories of the second class particle and the dual second class particle, the space-time paths will change in such a way that the flux $F(x, t)$ is increased by 1 for each $(x, t)$ within the trajectories. However, the first time the two trajectories meet (which happens with probability 1, according to Theorem 3.1), the two particles annihilate each other (since one corresponds to an extra source, whereas the other corresponds to an extra sink). This means that the shaded area in Figure 5, between the two trajectories, is the only area where the space-time paths are changed, and within this area the flux is increased by 1 .

Now we define the following "rainfall" process, which will emulate the adding of extra $\alpha$-points to a stationary Hammersley process. We define this process in $[0, \infty)^{3}$, where the first two variables $x$ and $t$ are the same as we have seen before, but the third parameter $\tau$ is a kind of external time parameter. Consider three independent Poisson processes in this space: a Poisson process of intensity 1 on the plane $[0, \infty) \times\{0\} \times[0, \infty)$, a Poisson process of intensity 1 on the plane $\{0\} \times[0, \infty) \times[0, \infty)$ and a Poisson process with intensity measure $2 \tau d x d t d \tau$ on $[0, \infty)^{3}$.

Then define $L^{(\tau)}$ as the Hammersley process at "time" $\tau$ that uses as its sources the projection of all Poisson points in $[0, \infty) \times\{0\} \times[0, \tau]$ onto the $x$-axis, as its sinks the projection of all Poisson points in $\{0\} \times[0, \infty) \times[0, \tau]$ onto the $t$-axis and as its $\alpha$-points, the projection of all Poisson points in $[0, \infty)^{2} \times[0, \tau]$ onto the $(x, t)$-plane. It is easy to check that $L^{(\tau)}$ will be a stationary Hammersley process with source and sink intensity equal to $\tau$, and the intensity of $\alpha$-points equal to $\tau^{2}$. The advantage of this construction lies in the fact that we have coupled a family of stationary Hammersley processes.

We will use this process to prove the following theorem.

Theorem 3.2 Consider a Hammersley process with intensity of sources and sinks being $\tau$ and intensity of $\alpha$-points being $\tau^{2}$. Let $O^{(\tau)}$ be the region in the plane enclosed by the trajectories 
$\left(X_{\tau}(t), t\right)$ and $\left(X_{\tau}^{\prime}(t), t\right)$ of a second class and a dual second class particle starting in the origin, until their first intersection point. Fix $x, t>0$ and let Area denote Lebesgue's measure on the plane. Then

$$
\mathbb{E A r e a}\left(O^{(\tau)} \cap[0, x] \times[0, t]\right)=\frac{x+t}{2 \tau}-\frac{1}{2 \tau}\left(\mathbb{E}\left(x-X_{\tau}(t)\right)_{+}+\mathbb{E}\left(t-X_{\tau}(x)\right)_{+}\right) .
$$

Proof: Consider the rainfall process described above and let $F_{\tau}(x, t)$ be the flux at the point $(x, t)$ of the stationary process $L^{(\tau)}$. To prove the theorem we will calculate the derivative

$$
\frac{\partial}{\partial \tau} \mathbb{E} F_{\tau}(x, t)
$$

in two different ways.

Take some small $d \tau>0$ and note that, since the processes $L^{(\tau)}$ and $L^{(\tau+d \tau)}$ are stationary, we can calculate the expected flux at both times directly:

$$
\mathbb{E} F_{\tau+d \tau}(x, t)-\mathbb{E} F_{\tau}(x, t)=x(\tau+d \tau)+t(\tau+d \tau)-x \tau-t \tau=(x+t) d \tau
$$

and therefore we get

$$
\frac{\partial}{\partial \tau} \mathbb{E} F_{\tau}(x, t)=x+t
$$

Now we consider a different way of calculating the difference

$$
\mathbb{E}\left(F_{\tau+d \tau}(x, t)-F_{\tau}(x, t)\right) .
$$

by using the coupling of $L^{(\tau)}$ and $L^{(\tau+d \tau)}$. Up to the first order of $d \tau$ we can assume that in the time interval $[\tau, \tau+d \tau]$ there either falls one extra source onto the segment $[0, x] \times\{0\}$, one extra sink onto the segment $\{0\} \times[0, t]$ or one extra $\alpha$-point inside the rectangle $(0, x] \times(0, t]$ (if nothing happens the difference is of course zero).

In the first case the flux will only be changed if the trajectory of a second class particle, starting at the dropped source on $[0, x] \times\{0\}$, will cross the line $[0, \infty) \times\{t\}$ to the left of the point $(x, t)$. In this case the flux will be increased by one. Similarly, in the second case the flux will increase by one only if the trajectory of a dual second class particle starting at the dropped sink on $\{0\} \times[0, t]$ will cross the line $\{x\} \times[0, \infty)$ to the right of the point $(x, t)$.

The area we are interested in arises from the third case. It follows from the explanation around Figure 5, that the flux at $(x, t)$ is increased by one only if $(x, t) \in O^{(\tau)}(u, v)$, where $(u, v)$ is the location of the extra $\alpha$-point and $O^{(\tau)}(u, v)$ denotes the area described in the theorem, but here the second class particle and the dual second class particle start at $(u, v)$.

Combining all three cases we have

$$
\begin{aligned}
\mathbb{E}\left(F_{\tau+d \tau}(x, t)-F_{\tau}(x, t)\right)= & d \tau \int_{0}^{x} P\left(X_{\tau, t}(u, 0)<x\right) d u+d \tau \int_{0}^{t} P\left(X_{\tau, t}^{\prime}(0, v)>x\right) d v+ \\
& +2 \tau d \tau \int_{0}^{x} \int_{0}^{t} P\left((x, t) \in O^{(\tau)}(u, v)\right) d u d v+o(d \tau),
\end{aligned}
$$


where $X_{\tau, t}(u, 0)$ and $X_{\tau, t}^{\prime}(0, v)$ are $x$-coordinates at time $t$ of a second class and a dual second class particle of $L^{(\tau)}$, starting at points $(u, 0)$ and $(0, v)$ respectively.

Using the fact that in the stationary Hammersley process $L^{(\tau)}, O^{(\tau)}$ and $O^{(\tau)}(u, v)$ have the same distribution modulo a translation, we can rewrite the last integral of (3.5) in the following way:

$$
\begin{gathered}
\int_{0}^{x} \int_{0}^{t} P\left((x, t) \in O^{(\tau)}(u, v)\right) d u d v=\int_{0}^{x} \int_{0}^{t} P\left((x-u, t-v) \in O^{(\tau)}\right) d u d v= \\
=\mathbb{E} \int_{0}^{x} \int_{0}^{t} \mathbf{1}_{O^{(\tau)}}(u, v) d u d v=\mathbb{E} \operatorname{Area}\left(O^{(\tau)} \cap[0, x] \times[0, t]\right) .
\end{gathered}
$$

Dividing both parts of (3.5) by $d \tau$, taking the limit $d \tau \rightarrow 0$ and using (3.3) we arrive at

$$
x+t=\int_{0}^{x} P\left(X_{\tau, t}(u, 0)<x\right) d u+\int_{0}^{t} P\left(X_{\tau, t}^{\prime}(0, v)>x\right) d v+2 \tau \mathbb{E} \operatorname{Area}\left(O^{(\tau)} \cap[0, x] \times[0, t]\right) .
$$

Again using the stationarity of $L^{(\tau)}$, we can see that

$$
P\left(X_{\tau, t}(u, 0)<x\right)=P\left(X_{\tau}(t)<x-u\right)
$$

and

$$
P\left(X_{\tau, t}^{\prime}(0, v)>x\right)=P\left(X_{\tau}^{\prime}(t-v)>x\right)
$$

This leads to

$$
\mathbb{E} \text { Area }\left(O_{\tau} \cap[0, x] \times[0, t]\right)=\frac{x+t}{2 \tau}-\frac{1}{2 \tau} \int_{0}^{x} P\left(X_{\tau}(t)<u\right) d u-\frac{1}{2 \tau} \int_{0}^{t} P\left(X_{\tau}^{\prime}(v)>x\right) d v .
$$

Consider the first integral on the right side of (3.6). We can rewrite it in the following way:

$$
\begin{aligned}
\int_{0}^{x} P\left(X_{\tau}(t)<u\right) d u & =\mathbb{E}\left(\int_{0}^{x} \mathbf{1}_{\left\{X_{\tau}(t)<u\right\}} d u\right) \\
& =\mathbb{E}\left(x-X_{\tau}(t)\right)_{+} .
\end{aligned}
$$

Using the equality $P\left(X_{\tau}^{\prime}(v)>x\right)=P\left(X_{\tau}(x)<v\right)$, which follows from the symmetry of the process $L^{(\tau)}$ (note that we have the same intensity of sources and sinks), we conclude a similar formula for the second integral in (3.6)

$$
\int_{0}^{t} P\left(X_{\tau}^{\prime}(v)>x\right) d v=\mathbb{E}\left(t-X_{\tau}(x)\right)_{+} .
$$

Combining these results we have proved that

$$
\mathbb{E} \operatorname{Area}\left(O^{(\tau)} \cap[0, x] \times[0, t]\right)=\frac{x+t}{2 \tau}-\frac{1}{2 \tau}\left(\mathbb{E}\left(x-X_{\tau}(t)\right)_{+}+\mathbb{E}\left(t-X_{\tau}(x)\right)_{+}\right) .
$$


This result is only valid for stationary Hammersley processes with equal source and sink intensity. However, if we consider $L_{\lambda}$, a stationary Hammersley process with source intensity $\lambda$, sink intensity $1 / \lambda$ and intensity of $\alpha$-points equal to 1 , it is clear that this process has the same distribution as the image of the process $L_{\lambda=1}$ under the map

$$
\Phi_{\lambda}(x, t)=(x / \lambda, \lambda t) .
$$

This observation easily leads to the following corollary.

Corollary 3.3 Consider a stationary Hammersley $L_{\lambda}$. Let $O_{\lambda}$ be the area in the plane enclosed by the trajectories $\left(X_{t}, t\right)$ and $\left(X_{t}^{\prime}, t\right)$ of a second class and a dual second class particle starting in the origin, until their first intersection point. Fix $x, t>0$ and let Area denote Lebesgue's measure on the plane. Then

$$
\mathbb{E} \text { Area }\left(O_{\lambda} \cap[0, x] \times[0, t]\right)=\frac{\lambda x+t / \lambda}{2}-\frac{\lambda}{2}\left(\mathbb{E}\left(x-X_{t}\right)_{+}+\mathbb{E}\left(t / \lambda^{2}-X_{\lambda^{2} x}\right)_{+}\right) .
$$

Another interesting asymptotic corollary to Theorem 3.2 is the following.

Corollary 3.4 Consider a stationary Hammersley process $L_{\lambda}$.

1. For any fixed $x_{0}>0$ we have

$$
\mathbb{E} \text { Area }\left(O_{\lambda} \cap\left[0, x_{0}\right] \times[0, \infty)\right)=\frac{\lambda x_{0}+\lambda \mathbb{E} X_{\lambda^{2} x_{0}}}{2} .
$$

2. For any fixed $t_{0}>0$ we have

$$
\mathbb{E} \text { Area }\left(O_{\lambda} \cap[0, \infty) \times\left[0, t_{0}\right]\right)=\frac{t_{0} / \lambda+\lambda \mathbb{E} X_{t_{0}}}{2} .
$$

3.

$$
\lim _{x \rightarrow \infty} \frac{\mathbb{E} \operatorname{Area}\left(O_{\lambda} \cap[0, x] \times\left[0, \lambda^{2} x\right]\right)}{x}=\lambda
$$

Proof: For the first statement, consider $\mathbb{E}\left(x-X_{t}\right)_{+}$. Note that

$$
\left(x-X_{t}\right)_{+} \rightarrow 0 \quad \text { a.s. }
$$

as $t \rightarrow \infty$. Since $\left(x-X_{t}\right)_{+}$is bounded by $x$, we get

$$
\mathbb{E}\left(x-X_{t}\right)_{+} \rightarrow 0 \quad(t \rightarrow \infty) .
$$

Now consider $t / \lambda^{2}-\mathbb{E}\left(t / \lambda^{2}-X_{\lambda^{2} x}\right)_{+}=\mathbb{E}\left(\min \left(X_{\lambda^{2} x}, t / \lambda^{2}\right)\right)$. Clearly,

$$
\mathbb{E}\left(\min \left(X_{\lambda^{2} x}, t / \lambda^{2}\right)\right) \rightarrow \mathbb{E} X_{\lambda^{2} x} \quad(t \rightarrow \infty),
$$

so the first statement is a direct consequence of these observations and Corollary 3.3. The proof of the second statement is completely analogous. 
The last statement follows from the fact that in $L_{\lambda}$, we have

$$
\frac{X_{\lambda^{2} x}}{x} \rightarrow 1 \quad \text { a.s. }
$$

Now we use dominated convergence to get the desired result from Corollary 3.3.

This last corollary seems to indicate that the two trajectories of the second class particle and the dual second class particle run parallel at average vertical distance $\lambda$. However, the true behavior of the two trajectories is that they often collide quite quickly, but with low probability they drift away from each other, in which case $O_{\lambda} \cap[0, x] \times\left[0, \lambda^{2} x\right]$ grows quadratically in $x$. It does follow from Theorem 3.1 that eventually the two trajectories will touch. We wish to remark that it is not hard, using the methods of [CATor and Groeneboom (2005)] and this paper, together with some crude estimates, to show that $\mathbb{E} X_{t}<+\infty$.

\section{References}

[Aldous and Diaconis (1995)] Aldous, D. And Diaconis, P. (1999). Hammersley's interacting particle process and longest increasing subsequences. Probab. Theory \& Related Fields 103, 199-213.

[Cator and Groeneboom (2005)] Cator, E. and Groeneboom, P. (2005). Hammersley's process with sources and sinks. Annals of Probability 33(3), 879-903.

[Colleti and Pimentel (2006)] Colleti C. and Pimentel, L.P.R. (2006). Beta-paths in the Hammersley process. (arXiv:math.PR/0603382)

[Ferrari and Kipnis (1995)] Ferrari, P.A. And Kipnis, C. (1995). Second class particles in the rarefaction front. Annales. de L'Institut Henri Poincaré, vol. 31 (1), 143-154.

[Groeneboom (2002)] Groeneboom, P. (2002). Hydrodynamical methods for analyzing longest increasing subsequences. Journal of Computational and Applied Mathematics, vol. 142, 83105.

[Hammersley (1972)] Hammersley, J.M. (1972). A few seedlings of research. Proc. 6th Berkeley Symp. Math. Statist. and Probability, vol. 1, 345-394.

[Liggett (1999)] Liggett, T.M. (1999). Stochastic Interacting Systems, Contact, Voter and Exclusion Processes. Springer, New York.

[Seppäläinen (2002)] SeppäLäınen, T. (2002). Diffusive fluctuations for one-dimensional totally asymmetric interacting random dynamics. Comm. Math. Phys. 229 , 141-182 